\title{
Erratum to: Building capacity to reduce health inequalities through health promotion in Europe
}

\author{
Lisa Gugglberger ${ }^{1,2} \cdot$ Nigel Sherriff $^{1} \cdot$ John Kenneth Davies ${ }^{1} \cdot$ Stephan Van den Broucke ${ }^{3}$
}

Published online: 27 May 2016

(C) Springer-Verlag Berlin Heidelberg 2016

Erratum to: J Public Health (2016) 24:73-81

DOI 10.1007/s10389-015-0699-y

In the published version of this article, the information regarding the Ethical approval for this study was withheld. It should say:

"Ethical approval for WP3 and WP5 of the project was received by the Faculty of Health and Social Science Research Ethics and Governance Committee at the University of Brighton."

The online version of the original article can be found at http://dx.doi.org/ 10.1007/s10389-015-0699-y.

Lisa Gugglberger

lisa.gugglberger@ifgp.at

1 Centre for Health Research (CHR), University of Brighton, Mayfield House, Falmer, Brighton BN1 9PH, UK

2 Ludwig Boltzmann Institute Health Promotion Research, Ludwig Boltzmann Gesellschaft, Untere Donaustraße 47/B09, 1020 Vienna, Austria

3 Psychological Sciences Research Institute, Université catholique de Louvain, 10 Place Cardinal Mercier,

B-1348 Louvain-la-Neuve, Belgium 